\title{
SARS-CoV-2 and ECMO: early results and experience
}

\author{
Waqas Akhtar $^{1}$ (D) $\cdot$ Olusegun Olusanya $^{1} \cdot$ Marta Montero Baladia ${ }^{1} \cdot$ Harriet Young ${ }^{1} \cdot$ Sachin Shah $^{1}$
}

Received: 7 August 2020 /Revised: 9 October 2020 / Accepted: 21 October 2020 / Published online: 24 November 2020

(C) Indian Association of Cardiovascular-Thoracic Surgeons 2020

\begin{abstract}
Introduction In this paper, we describe our experience and early outcomes with critically unwell severe acute respiratory syndrome coronavirus 2 (SARS-CoV-2) patients who required extracorporeal membrane oxygenation (ECMO). We present our standard practices around ECMO decision-making, retrieval, cannulation, ventilation, anticoagulation, tracheostomy, imaging and steroids.

Methods A retrospective cohort study using data from the hospital notes on all SARS-CoV-2 patients who required extracorporeal support at St Bartholomew's Hospital between 1 March 2020 and 31 July 2020. In total, this included 18 patients over this time period.

Results In total, 18 patients were managed with extracorporeal support and of these 14 survived (78\%) with 4 deaths (22\%). The mean duration from hospital admission to intubation was $4.1 \pm 3.4$ days, mean time from intubation to ECMO $2.3 \pm 2$ days and mean run on ECMO 17.7 \pm 9.4 days. Survivor mean days from intubation to extubation was $20.6 \pm 9.9$ days and survivor mean days from intubation to tracheostomy decannulation $46.6 \pm 15.3$ days. Time from hospital admission to discharge in survivors was a mean of $57.2 \pm 25.8$ days. Of the patients requiring extracorporeal support, the initial mode was veno-venous (VV) in 15 (83\%), veno-arterial (VA) in $2(11 \%)$ and veno-venous-arterial (VVA) in $1(6 \%)$. On VV extracorporeal support, 2 (11\%) required additional VVA. Renal replacement therapy was required in $10(56 \%)$ of the patients. Anticoagulation target anti-Xa of $0.2-0.4$ was set, with $10(56 \%)$ patients having a deep vein thrombosis or pulmonary embolism detected and $2(11 \%)$ patients suffering an intracranial haemorrhage. Tracheostomy was performed in $9(50 \%)$ of the patients and high-dose methylprednisolone was given to 7 (39\%) of the patients.

Conclusion In our cohort of patients with severe SARS-CoV-2 respiratory failure, a long period of invasive ventilation and extracorporeal support was required but achieving good outcomes despite this. There was a significant burden of thromboembolic disease and renal injury. A significant proportion of patients required tracheostomy and steroids to facilitate weaning.
\end{abstract}

Keywords SARS-CoV-2 $\cdot$ Coronavirus $\cdot$ ECMO $\cdot$ ARDS $\cdot$ Extracorporeal $\cdot$ Covid- 19

\section{Introduction}

Severe acute respiratory syndrome coronavirus 2 (SARS$\mathrm{CoV}-2$ ) is a positive sense single-stranded ribonucleic acid virus that is the causative agent for the ongoing coronavirus disease 2019 (COVID-19) pandemic [1]. Extracorporeal membrane oxygenation (ECMO) is an extracorporeal technique providing respiratory and cardiac support in selected patients with severe cardiac or respiratory failure. Initial reports on the use of ECMO in COVID-19 reported a very high

Sachin Shah

s.shah4@nhs.net

1 Department of Intensive Care, St Bartholomew's Hospital, London EC1A 7BE, UK mortality [2]; however, a growing body of evidence has challenged these initial findings $[3,4]$. In this article, we describe our experience with this technology in COVID-19 patients.

\section{SARS-CoV-2 clinical presentation}

After an initial incubation period of 5-6 days [5], people commonly present with initial symptoms of fever, cough, diarrhoea, fatigue, myalgia and anorexia [6]. Leukopaenia and raised inflammatory markers (ferritin, lactate dehydrogenase and C-reactive protein) are hallmarks of the disease [7]. Chest radiography, lung ultrasonography and computerised tomography show areas of change but are non-specific [8]. Diagnosis can be confirmed by reverse transcriptasepolymerase chain reaction of a nasal swab or tracheal aspirate [9]. A vast majority of patients develop mild disease and 
recover at home, with $4 \%$ of UK patients developing severe disease requiring admission to intensive care [10].

Severe disease is characterised by hypoxaemic respiratory failure [11], with most patients meeting the criteria for acute respiratory distress syndrome (ARDS). Other features include cardiomyopathy, acute kidney and/or liver injury, rhabdomyolysis, coagulopathy, cytokine release syndrome and central nervous system involvement. Risk factors for severe disease include advancing age, male sex, diabetes, hypertension and non-white ethnicity [12].

To date, UK intensive care units have admitted more than 10,000 patients, with a mean age of 59 years. In total, $60 \%$ of patients have gone on to require mechanical ventilation within the first $24 \mathrm{~h}$. Overall intensive care mortality is $40 \%$ with $26 \%$ of patients requiring renal replacement therapy [13].

\section{ECMO in the UK}

Following the 2009 Influenza A virus subtype H1N1 Pandemic, a national respiratory ECMO service was commissioned [14]. Expertise was concentrated in 5 centres:

- Royal Papworth Hospital, Cambridge

- The Royal Brompton Hospital, London

- St Thomas's Hospital, London

- Glenfield Hospital, Leicester

- The Wythenshawe Hospital, Manchester

A national pathway created allowing for timely referral and retrieval as required. These centres are staffed 24/7 with consultants skilled in the management of respiratory failure, and have mobile ECMO teams available that can be deployed to retrieve critically unwell patients across the country — by road or air as required.

In non-pandemic conditions, these centres have the capacity to care for between 25 and 30 patients. During the COVID19 pandemic, surge capacity was activated at each centre and two extra centres were added to the national framework; St Bartholomew's Hospital, London and the Freeman Hospital, Newcastle. At peak surge, the UK was capable of delivering ECMO to 100 patients nationwide [15]. By the end of May 2020, 216 patients have been placed on ECMO for COVID-19 nationally [15]. National inclusion criteria for ECMO, adapted during the pandemic, are shown in Table 1.

\section{St Bartholomew's Hospital (Barts) ECMO service}

The Barts ECMO service is based in Barts Heart Centre in London, the largest specialised cardiovascular centre in Europe. We provide both venous-venous (VV) and venoarterial (VA) ECMO service to hospitals within Barts and the London NHS Trust, the largest NHS trust within the UK. This encompasses 4 major hospitals with emergency departments and serves a population of 2.5 million [16]. The service is run by 8 consultants, who can cannulate $24 \mathrm{~h}$ a day in addition to several more supporting consultants who can manage patients on our 14-bed intensive care. Referrals are taken directly by the on-call consultant for ECMO and through a conference call multidisciplinary team for all VA ECMO cases. The core retrieval team consists of the ECMO consultant and cardiac perfusionist with additional space for a training fellow. A specially adapted transfer trolley with ventilator, syringe pumps and ECMO machine is transported via a repurposed ambulance along with all equipment required for cannulation to the referring hospital. The patient is brought back to Barts through the London Ambulance Service.

The pandemic stretched our services requiring an expansion from a single unit to four wards to cope with the influx of ventilated and critically unwell patients. The ECMO service reached the peak capacity of 10 patients on extracorporeal support. Many doctors and nurses from our cardiology and oncology teams transferred to intensive care for several weeks to assist with the extraordinary influx of critically ill patients.

\section{Methods}

This retrospective study included all patients from our institution who tested positive for SARS-COV2 via polymerase chain reaction testing and were placed on ECMO, from 1 March 2020 to 31 July 2020. Patients with SARS-COV2 who were not placed on ECMO were used as comparative controls. To be considered for ECMO, the patients had to satisfy the national criteria as per Table 1 .

\section{Table 1 NHS ECMO referral criteria}

NHS ECMO referral criteria

(taken from https://www.england.nhs.

uk/coronavirus/wp-content/uploads/sites/52/2020/04/

C0156-Extra-Corporeal-Membrane-Oxygenation-ECMO-

Adult-Speciality-Guide-1.pdf)

Inclusion criteria (updated June 2020)

Potentially reversible severe respiratory failure

Lung Injury Score $\geq 3$ or uncompensated hypercapnia

with a pH 7.20 or less

Failed trial of ventilation in prone positioning $\geq 6 \mathrm{~h}$

(unless contraindicated)

Failed optimal respiratory management/lung protective ventilation

Clinical Frailty Scale category $\leq 3$

If RESP Score $\leq 3$, ECMO should be considered only

after agreement across at least two centres

Exclusion criteria

Refractory multiorgan failure

Evidence of severe neurological injury 


\section{ECMO technical aspects}

Our standard approach for percutaneous cannulation is a bifemoral for both VV and VA ECMO. The second-line access point if one femoral access is not possible or a third cannula is needed is the internal jugular vein. If there are insufficient access points, then a bicaval dual lumen cannula can be placed in the internal jugular alone. The insertion of the cannula is through a standard Seldinger approach under linear ultrasound-guided puncture. Additionally to ensure the guidewire is within the correct vessel and maintains position, either echocardiography through a bedside transthoracic subcostal window, bedside transoesophageal bicaval and aortic views, or fluoroscopy in the cardiac catheter laboratory is utilised.

The standard ranges of cannula sizes utilised are as follows. Standard femoral venous multistage drainage is a 21-25French (Fr) cannula placed typically at $45-50 \mathrm{~cm}(\mathrm{~cm})$ length. Standard femoral venous return is a 21-25-Fr cannula placed typically at $50-55 \mathrm{~cm}$ length. Standard femoral arterial return is $15-17-F r$ cannula placed typically at $15-20 \mathrm{~cm}$. Standard 27-31-Fr bicaval dual-lumen cannula insertion into the internal jugular vein needs to be carefully positioned using fluoroscopy and transoesophageal echo due to importance of the position and direction of the return jet. The vessel utilised will ideally be twice the diameter of the cannula diameter to reduce risk of venous thrombosis. In the case of arterial access if insufficient blood flow is reaching the distal limb, then a 78-Fr return perfusion cannula is inserted to the common femoral artery via ultrasound or fluoroscopy guidance and ECMO blood flow diverted to the limb at 100-200 $\mathrm{ml} / \mathrm{min}$.

We utilise a mixture of Cardiohelp ${ }^{\mathrm{TM}}$ (Getinge, Sweden) and Levotronix CentrimagTM (Chalicemedical, UK) ECMO systems for our circuits. All retrievals are made on the Cardiohelp ${ }^{\mathrm{TM}}$ system due to ease of transfer. Patients requiring ECMO for longer durations are often placed on the Levotronix Centrimag ${ }^{\mathrm{TM}}$ system. The minimum standard blood flow is not lower than $2 \mathrm{~L} / \mathrm{min}$ to reduce risk of clot formation within the circuit. The blender fraction of inspired oxygen $\left(\mathrm{FiO}_{2}\right)$ sweep gas is always maintained at $1.00(100 \%)$ for VV ECMO and titrated according to arterial blood gas analysis for VA ECMO. The initial ventilator settings are designed to rest the lungs, typically pressure control ventilation with a positive end expiratory pressure of $10-15 \mathrm{~cm}$ of water $\left(\mathrm{cmH}_{2} 0\right)$ and peak inspiratory pressure of $20 \mathrm{cmH} 20$, respiratory rate $10-15$ breaths/min with $\mathrm{FiO}_{2} 0.3$ to 0.5 . Our protocol is to wean the sweep gas for VV ECMO and to leave the blood flow at a possible minimum level at all time. Decannulation is typically acceptable once the patient has been stable off sweep gas for a $24 \mathrm{~h}$ period. VA ECMO titration is more complex and involves reductions in flow with continuous cardiac assessment via pulmonary catheter and echocardiography.
Anticoagulation and renal replacement therapy

All patients on ECMO were anti-coagulated with unfractionated heparin to a mean target anti-Factor Xa (anti-Xa) level of $0.28 \mathrm{IU} /$ $\mathrm{mL}$. Our usual range is $0.2-0.4 \mathrm{IU} / \mathrm{mL}$ for $\mathrm{VV}$ and $0.3-0.5 \mathrm{IU} /$ $\mathrm{mL}$ for VA ECMO. Some patients had their target range extended to $0.4-0.8 \mathrm{IU} / \mathrm{mL}$ if there were also other indications for anticoagulation such as pulmonary embolism. Indeed $10(56 \%)$ of our patients on ECMO developed some form of venous thromboembolism, which reflected higher rates of pulmonary embolism and deep vein thrombosis found in the wider intensive care SARS-CoV-2 population. We felt anti-Xa provided a more reliable and consistent result compared to activated partial thromboplastin time ratio in our local laboratory service and this was checked at least once a day and more frequently while achieving a steady state. We had two intra-cerebral haemorrhage, both leading to death, and 2 cases of gastrointestinal haemorrhage, of which one required endoscopic intervention. We were able to run the ECMO circuit without anticoagulation for longer than a week without any issue in the patients with gastrointestinal and intra-cerebral haemorrhage; this is a recognised strategy in ECMO patients at high risk of bleeding [17].

A high proportion of ventilated patients with SARS-CoV-2 required renal replacement therapy, in the ECMO cohort reaching $10(56 \%)$. We believed part of this to be the disease process but also with many having elevated sodium levels a possible reflection of intravascular fluid status. As such, we changed our daily fluid balance targets from the usual negative balances to a more neutral or slightly positive daily balance to pre-empt any pre-renal failure. Many patients within the ventilated SARS-CoV-2 cohort clotted off renal replacement circuits within hours of initiation of therapy despite good access. This did not occur within the ECMO cohort and it was felt to be due to the standard anticoagulation. Indeed, as the pandemic progressed, all ventilated patients on renal replacement therapy were managed with heparin anticoagulation and the issue subsided. A hospital-wide protocol was established for higher prophylactic low molecular weight heparin doses based on a Ddimer cutoff of $3 \mu \mathrm{g} / \mathrm{mL}$ as higher levels were thought to reflect increased coagulability. However, higher D-dimer levels along with consumption of fibrinogen are used as signs of circuit failure within ECMO and therefore less interpretable.

\section{Airway and ventilation}

As a standard, we aimed to manage all ventilated patients with SARS-CoV-2 with lung protective ventilation at $6 \mathrm{~mL} / \mathrm{kg}$, paralysis, proning and trial of pulmonary vasodilators prior to initiation of ECMO unless the clinical situation was in extremis. In our cohort, 16 (89\%) patients received proning prior to ECMO with the exceptions being two cases of myocarditis felt to be too unstable to turn. As previously described, the initial difficulty was predominantly of oxygenation with maintenance of 
adequate compliance. However, often within the first week of the ECMO run, patients would often develop compliance below $10 \mathrm{~mL} / \mathrm{cmH}_{2} 0$ and effectively dead space ventilation. This was found to be due to a mixture of issues including dense widespread infiltration of the lung parenchyma, heavy secretion burden with mucus plugging or collapse of lung field from endotracheal tube migration. The latter was often associated with large airways that appeared oedematous with loss of ring cartilage definition and marked tracheomalacia. Indeed some patients if transitioned to a pressure support ventilation mode developed obstructive breathing patterns due to occlusion of the endotracheal tube tip that was overcome by mandatory ventilation modes.

As an initial concern for aerosolisation of SARS-CoV-2, most ventilators were run with dry circuits. However, this led to repeated condensation obstruction of heat and moister exchange filters that was seen in other intensive cares in the UK [18]. As such we switched to humidified circuits as normal practice and this was not observed as often. The use of ECMO provided safety with regard to bronchoscopy, and when required patients underwent fibreoptic bronchoscopy for bronchoalveolar lavage and mucus clearance with no concurrent ventilation to reduce risk to staff.

Nine $(50 \%)$ of the ECMO patients required tracheostomy, which carried a higher than usual complication rate for our centre felt to be due to the abnormal airways described above. The procedure was carried out with specialised ventilated hoods and in a theatre environment to provide further protection from coronavirus

\section{Cardiovascular management}

All patients had central venous catheters and arterial lines placed. Inotropes and vasopressors were used as needed to maintain a mean arterial pressure (MAP) of at least $65 \mathrm{mmHg}$. Our first-line vasopressor was noradrenaline, and our first-line inotrope was milrinone.

In VA ECMO patients, a pulmonary artery catheter (PAC) was inserted to provide continuous cardiac output measurements, and to guide weaning. Echocardiography was also performed daily to assess cardiac dimensions, valvulopathy and readiness for weaning. In VV ECMO patients, cardiac output monitoring was not routinely used; if required, oesophageal Doppler monitoring or echocardiography was performed. Comprehensive echocardiography was performed on the day of admission to the ECMO ICU to assess cannula position and biventricular function, and was repeated when clinically indicated (new signs of shock or cannula repositioning).

\section{Neurological management}

All patients had hourly assessments at the bedside of neurological function, in the form of the Richmond Agitation and
Sedation Score (RASS) and pupillary assessment. While sedated and on ECMO, the target RASS was - 4, and this was achieved using propofol and fentanyl as first-line agents. Neuromuscular blockade was provided with either atracurium or vecuronium, and was targeted to full muscle relaxation using train of four monitoring. If the patient was awake and spontaneously ventilating, a Confusion Assessment Method in Intensive Care (CAM-ICU) score was performed once a day. A full neurological examination was performed twice a day during the ward rounds by the medical teams.

\section{Gastrointestinal and nutrition management}

Patients were enterally fed via a nasogastric or orogastric route as first line. Orogastric tubes were often chosen over nasogastric tubes in patients established on ECMO who were fully anticoagulated, in order to avoid significant epistaxis. Jejunal feeding or total parenteral nutrition was provided in cases of feeding failure; this was undertaken under the leadership of a critical care dietitian who reviewed patients daily.

\section{Imaging}

Our previous experience has shown that computed tomography $(\mathrm{CT})$ scanning is safe and feasible for patients on ECMO [19]. However, this data also showed that the effect of lung or abdominal scanning was modest on clinical decision-making. Furthermore with a risk to staff from exposure to SARS-CoV2 increased by the movement of these patients in the hospital, we made a decision to not routinely scan our SARS-CoV-2 ECMO population. We utilised chest radiographs and lung ultrasound to provide the majority of diagnostic chest imaging and proceeded to $\mathrm{CT}$ when clinically indicated. Clinical indications for $\mathrm{CT}$ were:

- Suspected neurological complications, based on clinical examination — particularly pupillary abnormalities

- Interval chest CT performed at 7-10 days in individuals with no clinical recovery, looking for organising pneumonia

- CT angiography in patients with acute gastrointestinal or pulmonary haemorrhage

Our use of echocardiography has been described above.

\section{Laboratory tests}

The patients' full blood count, urea and electrolyte profile, liver function, bone profile and C-reactive profile were tested daily. Heparinised patients had 4 hourly measurements of anti-Xa levels and heparin levels adjusted accordingly until a steady state achieved and then repeated every $24 \mathrm{~h}$. Ferritin and procalcitonin levels were tested twice weekly. 


\section{Antimicrobial and steroids}

As a standard, all SARS-CoV-2 patients were given treatment with Co-amoxiclav, clarithromycin and oseltamivir (stopped after negative respiratory swab for Influenza) on admission. After completing a 5-7-day course of these antibiotics, further treatment was only instigated if there was clinical, laboratory or radiological indication of a bacterial super-infection. We found that $\mathrm{C}$-reactive protein remained elevated regardless of the clinical status but that procalcitonin appeared to follow the clinical picture of a bacterial super-infection. We found many patients developed similar gram-ve bacterial super-infections, such as Klebsiella pneumonia, even if admitted from remote hospitals within our network and managed on separate wards.

Based on previous experience with the H1N1 pandemic, the team decided to give pulsed methylprednisolone to patients with SARS-CoV-2 who failed to progress despite 2 weeks on ECMO and lung protective ventilation. This was delivered as a pulse of $500 \mathrm{mg}-1$ $\mathrm{gm}$ for 3 days followed by an adapted regimen of Meduri et al. [20]; $2 \mathrm{mg} / \mathrm{kg} /$ day from days 4 to 7 , $1 \mathrm{mg} / \mathrm{kg} /$ day from days 7 to 14 . In our cohort, 7 (39\%) patients received high-dose methylprednisolone on an average 14.1 days post intubation.

\section{Results}

Table 2 provides demographic data of all patients admitted to our intensive care unit with a total of 79 SARS-CoV-2-positive patients and a survival rate of $70 \%$ as of 5 June 2020. This compares favourably with the national survival rate of $57.4 \%$. There were significant differences in our cohort to the national average, with a higher proportion of men and ethnic minorities.

In total, we managed 18 patients with SARS-CoV-2 on ECMO during the pandemic. Their data is displayed in Table 3. The cohort was relatively young with a mean age of $47.3 \pm 9.8$ years and $89 \%$ male preponderance. Black and Asian cohorts accounted for $78 \%$ of the ECMO patients [14]. Those with hypertension and diabetes accounted for over half of all patients. The average time of hospital admission to intubation was $4.1 \pm 3.4$ days. The average time between intubation to subsequent ECMO was $2.3 \pm 2$ days. The Acute Physiology and Chronic Health Evaluation II (APACHE II) score for our ECMO patients was 12.2 compared to national general critical care population of 14.9 [11]. The survival rate (Table 4) of patients in this cohort was 14 (78\%). Of the four deaths seen, two were due to intracranial haemorrhage and two had life-sustaining therapy withdrawn due to progressive multi-organ failure. The deaths occurred on average $23.8 \pm 12.7$ days after admission. Neither of the patients with intracranial haemorrhage had any
Table 2 Demographic data for all intensive care patients with SARSCoV-2. A local summary and comparison to national data provided by the ICNARC report on SARS-CoV-2 in Critical Care (05 June 2020) [14]. Table represents SARS-CoV-2-positive patients only

St Bart's Hospital National (ICNARC)

\begin{tabular}{lcc}
\hline Age at admission (mean (SD)) & $57.4(12.8)$ & $58.8(12.6)$ \\
Sex $(n(\%))$ & $18(20.2)$ & $2805(29.2)$ \\
Female & $71(79.8)$ & $6814(70.8)$ \\
Male & & \\
Ethnicity $(n(\%))$ & $26(29.2)$ & $5882(67.0)$ \\
White & $1(1.1)$ & $151(1.7)$ \\
Mixed & $34(38.2)$ & $1316(15.0)$ \\
Asian & $23(25.8)$ & $852(9.7)$ \\
Black & $5(5.6)$ & $573(6.5)$ \\
Other & $15.7(5.6)$ & $14.9(5.3)$ \\
APACHE II Score & $55(69.6)$ & $4918(57.6)$ \\
Mean (SD) & $24(30.4)$ & $3615(42.4)$ \\
Outcome at end of critical care $(n(\%))$ & \\
Alive & $10(3.9,16)$ & $9(5,15)$ \\
Dead & & \\
Length of stay in critical care (days), median (IQR) (figures include \\
ECMO patients) \\
Survivors \\
Non-survivors \\
\hline
\end{tabular}

significant abnormalities in their coagulation profile, and an $11 \%$ intracranial haemorrhage prevalence is consistent with previous data [21].

Table 3 Patient characteristics in ECMO SARS-CoV-2-positive cohort 1st March- 31st July $2020(n=18)$

\begin{tabular}{ll}
\hline Characteristic & Value \\
\hline Age (years, mean \pm SD) & $47.3 \pm 9.8$ \\
Female sex (no. (\%)) & $2(11)$ \\
Body mass index (mean \pm SD) & $31 \pm 6.6$ \\
Ethnicity & \\
White (no. (\%)) & $4(22)$ \\
Black (no. (\%)) & $6(33)$ \\
Asian (no. (\%)) & $8(44)$ \\
Hypertension (no. $(\%))$ & $10(56)$ \\
Diabetes (no. (\%)) & $10(56)$ \\
Scores & \\
APACHE II (mean \pm SD) & $12.2 \pm 4$ \\
Frailty Score (mean \pm SD) & $1.8 \pm 0.5$ \\
RESP Score (mean \pm SD) & $4.4 \pm 1.1$ \\
Hospital admission to intubation (days, mean \pm SD) & $4.1 \pm 3.4$ \\
Intubation to ECMO (days, mean \pm SD) & $2.3 \pm 2$ \\
Proning pre ECMO (no. $(\%))$ ) & $16(89)$
\end{tabular}

SARS-CoV-2 positive determined by swab or tracheal aspirate sample; $n / n o$., number; $S D$, standard deviation; \%, percentage 


\section{Discussion}

In our small cohort of SARS-COV-2 ECMO patients, we have observed a survival rate of $78 \%$ despite initial reports suggesting a lack of efficacy. This is similar to results from two further published cohorts from Paris and North America [3, 4].

Our study has some unique features-our cannulation was performed entirely by intensivists, mostly at the bedside using transthoracic echocardiography guidance, and was mostly femoro-femoral. Our cohort was slightly older than the American or French groups with similarly positive outcomes.

We also observed several divergences in the management of patients with coronavirus on ECMO compared to our previous experiences. The profound hypoxia seen in patients infected with coronavirus along with a marked inflammatory response often meant very high blood flows were required within $\mathrm{VV}$ ECMO to maintain adequate tissue oxygenation. Six (33\%) of our patients required additional drainage cannulas to meet blood flows at times up to $8 \mathrm{~L} / \mathrm{min}$. The maximum flow achievable with a single cannula is usually around $5 \mathrm{~L} / \mathrm{min}$ before suctioning events precluded further increase in flow.

Five $(28 \%)$ of patients with profound shock thought to be a SARS-CoV-2 myocarditis required an arterial return cannula.
Of these, 2 required initial veno-arterial ECMO, 1 required veno-venous-arterial (VVA) ECMO, and 2 on VV required conversion to VVA ECMO. The oxygenators we utilise are rated for $8-10 \mathrm{~L} / \mathrm{min}$ of flow but can struggle to function with a flow rate above $7 \mathrm{~L} / \mathrm{min}$ and in two cases an additional oxygenator was added into the circuit due to continuing hypoxia with high ECMO flows. This achieved a small improvement in oxygen saturation levels in both patients.

The average length of ECMO run was longer than we had previously seen for ARDS with an average run of $17.7 \pm 9.4$ days. During this time, 5 (28\%) patients required a single circuit change due to rising trans-membrane pressures. There were no emergency ECMO complications such as pump failure or air entrainment in our cohort and no unanticipated cardiac arrests.

We empirically gave steroids prior to the publication of the RECOVERY trial [22] purely based on local expert opinion, and only in cases of refractory respiratory failure. The publication of RECOVERY provided some validation to our steroid use, and we have changed our unit protocols for managing COVID patients appropriately.

Despite close monitoring of anticoagulation, 2 patients developed intracranial haemorrhages which were rapidly detected, but were sadly too extensive for neurosurgical intervention. Both patients lost brainstem reflexes and were palliated.

Table 4 ECMO SARS-CoV-2 cohort 1 March-31 July 2020 outcomes $(n=18)$

\begin{tabular}{|c|c|}
\hline & Outcome \\
\hline Death (no. $(\%)$ ) & $4(22)$ \\
\hline Time from hospital admission to death (days, mean \pm SD) & $23.8 \pm 12.7$ \\
\hline Initial venous-venous ECMO (no. (\%)) & $15(83)$ \\
\hline Initial venous-arterial ECMO (no. (\%)) & $2(11)$ \\
\hline Initial veno-venous-arterial (VVA) ECMO (no. (\%)) & $1(6)$ \\
\hline Conversion to VVA ECMO (no. (\%)) & $2(11)$ \\
\hline Length of ECMO run (days, mean $\pm \mathrm{SD}$ ) & $17.7 \pm 9.4$ \\
\hline Required second drainage cannula (no. $(\%)$ ) & $6(33)$ \\
\hline Circuit change required (no. $(\%)$ ) & $5(28)$ \\
\hline Time from intubation to removal of endotracheal tube in survivors (days, mean $\pm \mathrm{SD}$ ) & $20.6 \pm 9.9$ \\
\hline Tracheostomy (no. (\%)) & $9(50)$ \\
\hline Time from intubation to tracheostomy (days, mean $\pm \mathrm{SD}$ ) & $35.3 \pm 9.5$ \\
\hline Time from intubation to tracheostomy decannulation in survivors (days, mean $\pm \mathrm{SD}$ ) & $46.6 \pm 15.3$ \\
\hline Anti-Xa target (mean $\pm \mathrm{SD})$ & $0.28 \pm 0.01$ \\
\hline Intracranial haemorrhage (no. (\%)) & $2(11)$ \\
\hline Gastrointestinal haemorrhage (no. (\%)) & $2(11)$ \\
\hline DVT/PE on CTPA or US Doppler (no. $(\%)$ ) & $10(56)$ \\
\hline High-dose methylprednisolone (no. (\%)) & $7(39)$ \\
\hline Intubation to methylprednisolone dose (days, mean $\pm \mathrm{SD}$ ) & $14.1 \pm 7.3$ \\
\hline Renal replacement therapy (no. $(\%)$ ) & $10(56)$ \\
\hline Time from hospital admission to discharge alive (days, mean $\pm \mathrm{SD}$ ) & $57.2 \pm 25.8^{*}$ \\
\hline
\end{tabular}

n/no., number; $S D$, standard deviation; $\%$, percentage

*Excludes one patient at day 75 inpatient as of 31 July 2020 
Two of our patients developed refractory multiorgan failureone while still on ECMO support, while the other was successfully decannulated but developed refractory pulmonary fibrosis and resultant pulmonary hypertension. There were no significant features at presentation that would have led us to withhold ECMO support in either of these patients. Further analysis into these patients is ongoing at local and national levels.

Our study has multiple limitations. It is a single-centre study with a small and highly selected cohort of patients, limiting its external validity. Large parts of our protocol were based on usual practice and expert opinion. Nonetheless, we are hopeful that other units may find our experience helpful.

\section{Conclusions}

The SARS-CoV-2 epidemic stretched our hospital capacity and staff to an unprecedented level the National Health Service has not seen previously. Despite initial discouraging reports of ECMO use, our group persevered and have demonstrated acceptable outcomes comparable to other international experience.

Other specific aspects of our experience have been longerthan-normal periods of ventilation and ECMO support, a high prevalence of tracheomalacia (leading to difficulties with ventilator weaning and tracheostomy insertion) and seeming success with steroid use prior to widespread adoption.

There are several points in these patients' care where traditionally withdrawal may have been considered but persistence appears to be the key message in SARS-CoV-2 with many making recoveries after lengthy critical care stays.

Acknowledgements Dr. Anthony Bastin, Dr. Jeremy Cordingley, Dr. Simon Finney, Prof Mark Griffiths, Dr. Victoria Heaviside, Dr. Hazem Lashin, Dr. Alastair Proudfoot, Dr. Stephen Shepherd, Dr. Ben Singer, Dr. Rosalba Spiritoso, Dr. Mary White.

Data availability On request by email

\section{Compliance with ethical standards}

Conflict of interest The authors declare that they have no conflict of interest.

Informed consent Waived due to the retrospective nature of this study as per our institutional protocol.

Human and animal rights None infringed in this study.

Ethics committee approval Waived due to the retrospective nature of the study.

\section{References}

1. Zhou F, Yu T, Du R, et al. Clinical course and risk factors for mortality of adult inpatients with COVID-19 in Wuhan, China: a retrospective cohort study. Lancet. 2020;395:1054-1062.https:// doi.org/10.1016/S0140-6736(20)30566-3.

2. Yang $\mathrm{X}, \mathrm{Yu} \mathrm{Y}, \mathrm{Xu}$ J, et al. Clinical course and outcomes of critically ill patients with SARS-CoV-2 pneumonia in Wuhan, China: a single-centered, retrospective, observational study. Lancet Respir Med. 2020;8:475-481. https://doi.org/10.1016/S2213-2600(20) 30079-5.

3. Schmidt M, Hajage D, Lebreton G, et al. Extracorporeal membrane oxygenation for severe acute respiratory distress syndrome associated with COVID-19: a retrospective cohort study [published online ahead of print, 2020 Aug 13]. Lancet Respir Med. 2020;S2213-2600(20):30328-3. https://doi.org/10.1016/S22132600(20)30328-3.

4. Mustafa AK, Alexander PJ, Joshi DJ, et al. Extracorporeal membrane oxygenation for patients with COVID-19 in severe respiratory failure. JAMA Surg. Published online August. 2020;11:990. https://doi.org/10.1001/jamasurg.2020.3950.

5. Gandhi RT, Lynch JB, Del Rio C. Mild or moderate COVID-19 [published online ahead of print, 2020 Apr 24]. N Engl J Med. 2020. https://doi.org/10.1056/NEJMcp2009249.

6. Chen N, Zhou M, Dong X, et al. Epidemiological and clinical characteristics of 99 cases of 2019 novel coronavirus pneumonia in Wuhan, China: a descriptive study. Lancet. 2020;395:507-513. https://doi.org/10.1016/S0140-6736(20)30211-7.

7. Wiersinga WJ, Rhodes A, Cheng AC, Peacock SJ, Prescott HC. Pathophysiology, transmission, diagnosis, and treatment of coronavirus disease 2019 (COVID-19): a review [published online ahead of print, $2020 \mathrm{Jul}$ 10]. JAMA. 2020. https://doi.org/10.1001/jama. 2020.12839.

8. Lomoro P, Verde F, Zerboni F, et al. COVID-19 pneumonia manifestations at the admission on chest ultrasound, radiographs, and CT: single-center study and comprehensive radiologic literature review. Eur J Radiol Open. 2020;7:100231. https://doi.org/10. 1016/j.ejro.2020.100231.

9. Bustin SA, Nolan T. RT-qPCR testing of SARS-CoV-2: a primer. Int J Mol Sci. 2020;21:3004.

10. Docherty AB, Harrison EM, Green CA, et al. Features of 20133 UK patients in hospital with covid-19 using the ISARIC WHO Clinical Characterisation Protocol: prospective observational cohort study. BMJ. 2020;369:m1985. Published 2020 May 22. https://doi. org/10.1136/bmj.m1985.

11. Berlin DA, Gulick RM, Martinez FJ. Severe COVID-19 [published online ahead of print, 2020 May 15]. N Engl J Med. 2020. https:// doi.org/10.1056/NEJMcp2009575.

12. Esai Selvan M. Risk factors for death from COVID-19. Nat Rev Immunol. 2020;20:407. https://doi.org/10.1038/s41577-020-03510 .

13. Intensive Care national audit and research centre. ICNARC report on COVID-19 in critical care. England. Intensive Care national audit and research centre. 2020 June 5 [cited 2020 June 5] Available at https://www.icnarc.org/Our-Audit/Audits/Cmp/ Reports (last checked 2/08/2020)

14. Specialised Commissioning Team NHS England. Clinical Commissioning Policy: Extra corporeal membrane oxygenation (ECMO) service for adults with cardiac failure 16028/P. England. NHS England. 2016 July 13 [Cited 2020 August 2]. Available from: https://www.england.nhs.uk/wp-content/uploads/2018/07/ Extra-corporeal-membrane-oxygenation-service-for-adults-withcardiac-failure.pdf

15. Warren A, Camporota L, Vuylsteke A. Surge capacity and updated admission criteria: response of the NHS-commissioned national respiratory extracorporeal membrane oxygenation network to the COVID-19 pandemic. Br J Anaesth. 2020; https://doi.org/10.1016/ j.bja.2020.06.008. 
16. Barts' Health NHS Trust. About us. England. Barts Health NHS Trust. 2020 [cited 2020 August 2]. Available from: https://www. bartshealth.nhs.uk/about-us.

17. Kurihara C, Walter JM, Karim A, et al. Feasibility of venovenous extracorporeal membrane oxygenation without systemic anticoagulation. Ann Thor Surg. 2020; https://doi.org/10.1016/j. athoracsur.2020.02.011.

18. Faculty of Intensive Care Medicine. ViRUS 1: Poor ventilation occurred in patients who were mechanically ventilated using a dry circuit with HME filters in place between catheter mount and rest of circuit, due to clogging in the HME filter. England. Faculty of Intensive Care Medicine. 2020 April 1 [cited 2020 August 2] https://www.ficm.ac.uk/sites/ default/files/virus1_0.pdf.

19. Richmond KM, Warburton KG, Finney SJ, Shah S, Reddi BAJ. Routine CT scanning of patients retrieved to a tertiary centre on veno-venous extracorporeal membrane oxygenation: a retrospective risk benefit analysis. Perfusion. 2018;33:438-444. https://doi.org/10.1177/0267659118763266.

20. Meduri GU, Golden E, Freire AX, et al. Methylprednisolone infusion in early severe ARDS: results of a randomized controlled trial. Chest. 2007;131:954-963. https://doi.org/10.1378/chest.06-2100.

21. Fletcher-Sandersjöö A, Thelin EP, Bartek J Jr, et al. Incidence, outcome, and predictors of intracranial hemorrhage in adult patients on extracorporeal membrane oxygenation: a systematic and narrative review. Front Neurol. 2018;9:548. https://doi.org/10.3389/ fneur.2018.00548.

22. The RECOVERY collaborative group. Dexamethasone in hospitalised patients with COVID-19- preliminary report. New England Journal of Medicine (online First) July 17. 2020. https:// doi.org/10.1056/NEJMoa2021436.

Publisher's note Springer Nature remains neutral with regard to jurisdictional claims in published maps and institutional affiliations. 